\title{
INFLUENCE OF BLUR ON FEATURE MATCHING AND A GEOMETRIC APPROACH FOR PHOTOGRAMMETRIC DEBLURRING
}

\author{
T. Sieberth ${ }^{\mathrm{a}, *}$, R. Wackrow ${ }^{\mathrm{a}}$, J. H. Chandler ${ }^{\mathrm{a}}$ \\ ${ }^{\text {a }}$ Loughborough University, School of Civil and Building Engineering, Leicestershire, LE11 3TU, United Kingdom - (T.Sieberth, \\ R.Wackrow, J.H.Chandler)@lboro.ac.uk
}

KEY WORDS: Blur, Computer, Vision, Feature, Matching, Photogrammetry, UAV

\begin{abstract}
:
Unmanned aerial vehicles (UAV) have become an interesting and active research topic for photogrammetry. Current research is based on images acquired by a UAV, which have a high ground resolution and good spectral and radiometric resolution, due to the low flight altitudes combined with a high resolution camera. UAV image flights are also cost efficient and have become attractive for many applications including change detection in small scale areas.
\end{abstract}

One of the main problems preventing full automation of data processing of UAV imagery is the degradation effect of blur caused by camera movement during image acquisition. This can be caused by the normal flight movement of the UAV as well as strong winds, turbulence or sudden operator inputs. This blur disturbs the visual analysis and interpretation of the data, causes errors and can degrade the accuracy in automatic photogrammetric processing algorithms.

The aim of this research is to develop a blur correction method to deblur UAV images. Deblurring of images is a widely researched topic and often based on the Wiener or Richardson-Lucy deconvolution, which require precise knowledge of both the blur path and extent. Even with knowledge about the blur kernel, the correction causes errors such as ringing, and the deblurred image appears "muddy" and not completely sharp. In the study reported in this paper, overlapping images are used to support the deblurring process, which is advantageous. An algorithm based on the Fourier transformation is presented. This works well in flat areas, but the need for geometrically correct sharp images may limit the application. Deblurring images needs to focus on geometric correct deblurring to assure geometric correct measurements.

\section{INTRODUCTION}

Constraints enforced on the acquisition of photographs for photogrammetry normally include a stable camera position and a stationary object. Unfortunately, lightweight small scale unmanned aerial vehicles (UAV) rarely provide a stable camera position. UAVs are easily affected by wind, gusts, turbulence or sudden operator inputs. However, their good manoeuvrability and flight path control combined with endurance, flight range and low cost make UAVs applicable for a range of different tasks (Eisenbeiß, 2009). The limited payload, regulatory restrictions and vulnerability of the UAV platforms encourage the use of low cost sensors, which often dictates the use of consumer grade cameras (Eisenbeiß, 2011). Unfortunately, the problem remains that the high spatial resolution of an image is often degraded due to motion blur. Since optical blur can be reduced using automatic focusing methods, motion blur remains a challenge. Resolving motion blur is an important and often researched topic in signal and image processing. The focus of many deblurring methods is often on spectral, radiometrical and geometrical deconvolution, which requires extensive calculations.

\subsection{Aims and Objectives}

Photogrammetry is defined as finding 'position, orientation, shape and size of objects from pictures' (Kraus, 2007). This is achieved by measuring coordinates of objects appearing in images. These measurements are mostly based on edge detection in the images because edges can often be detected and identified in overlapping images. In blurred images edges can disappear, change their position or become difficult to identify due to the degrading effect of motion blur (Fig 1).

Most methods use one blur kernel which represents the camera path during image acquisition. Using this general blur kernel for all pixels in the image is inaccurate because objects with a short camera to object distance are blurred more than objects further away. Also sensor rotation can change the blur kernel as the kernel is shorter for objects close to the rotation axis and larger

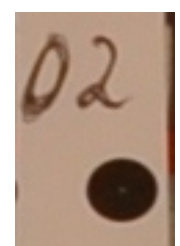

(a)

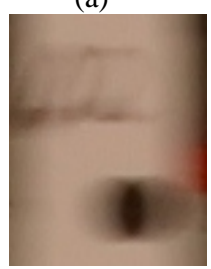

(c)

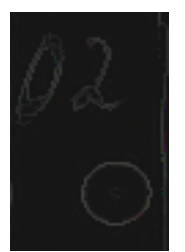

(b)

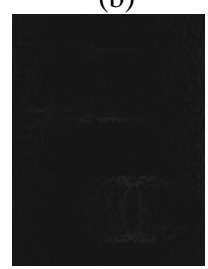

(d)
Figure 1. Example of a photogrammetric target. (a) Sharp image without any camera movement during image exposure. (b) The target is easy identifiable due to the well-defined edge between black and white. (c) Image blurred due to motion during image exposure. (d) The target is difficult to identify due to the difficulty to define the target outline.

\footnotetext{
* Corresponding author.
} 
for objects far away from the rotation axis. This needs to be considered for generating a precise deblurring method capable of working in $3 \mathrm{D}$.

This paper aims to analyse how much blur influences the detection of image overlap using image processing. Furthermore, image deblurring approaches suitable for photogrammetric purposes will be examined and discussed.

\section{RELATED WORK}

Aerial photogrammetry uses object information in images acquired by airborne platforms ranging from aircrafts to balloons. A platform recently developed and increasingly popular for image flights are lightweight UAVs, which are vulnerable to gusts, turbulence and are sensitive to operator inputs.

For a successful image flight it is necessary to carefully prepare the flight plan beforehand. To provide appropriate image geometry for 3D measurements a recommended image overlap of $60 \%$ along track and $20 \%$ across track should be used (Kraus 2004, Luhmann 2006). To calculate precise 3D coordinates for an object point, it is necessary to precisely measure the image coordinates in at least two images (Kraus, 2004). Additionally the exterior orientation and interior parameter of the camera are required. These parameters are required to fulfil the collinearity equation, which enables the 3D coordinates of points to be determined (Luhmann, 2006).

To carry out point measurements in multiple images automatically, feature points (signalised and unsignalised) need to be identified across frames. This is today based on the ScaleInvariant Feature Transform (SIFT), Speed-Up Robust Features (SURF) or similar algorithms. These algorithms detect suitable candidate features in the images, which are invariant to scaling, translation and rotation (Lowe, 2004, Bay et al., 2006). Similar features detected in two overlapping images can be referenced between them using least squares matching or other similar methods (Brown, 1992). Due to blur the same features can appear differently in the images and matching these becomes increasingly difficult. Even measuring well defined targets using automatic measurement methods and matching them between two images is influenced or even impossible (Sieberth et al., unpublished). These difficulties suggest the need to explain the influence of blur in photogrammetric processes.

There are various methods for image deblurring which have been published over the last decades. Image deblurring approaches can be separated into blind and non-blind deconvolution. Non-blind image deconvolution uses a-priori knowledge about the blur in the image. Blind deconvolution has only the blurred image and no additional information but the task of deriving a sharp image remains. Wiener deconvolution (Wiener, 1949) and Richardson-Lucy deconvolution (Richardson, 1972, Lucy, 1974) are blind deconvolution methods proposed decades ago and remain popular because they are both simple and efficient. A significant problem that occurs using these methods are ringing artefacts at steep edges. More advanced methods are often based on a probability calculation, which is also used by Wiener and Richardson-Lucy deconvolution. They aim to improve deblurring and the capability to work reliably, even with noise in the blurred image (Shan et al, 2008).

Non-blind image deconvolution methods can be carried out in various ways and requiring additional knowledge. The additional information can be gained through other overlapping images (Agrawal, 2012), precise IMU measurements (Joshi, 2008), video cameras (Tai et al., 2008), fluttering shutters (Raskar et al., 2006) or colour channel dependent exposure times (Lelégard, 2010). The main aim of these methods is to establish an appropriate blur kernel, which can be used by established deblurring algorithms.

\section{METHOD DEVELOPMENT}

\subsection{Feature Detection}

To analyse how critical motion blur influences feature detection, images with precisely known blur were generated. Blurred images were acquired using a Nikon D80 SLR camera, and a shaking table using a methodology described more fully in a paper by Sieberth et al. (2013). Briefly, this involved generating four images with different camera displacements each of between 0 to $1 \mathrm{~mm}$. Then these images were processed using the SURF function provided by OpenCV (SurfFeatureDetector), to detect feature points in the images (OpenCV, 2014). In the following step a "brute force matching method" (BFMatcher) was used to connect detected feature points. The connection was made between the feature points in the sharp image and each of the blurred images. The connection lines between sharp and blurred image should be parallel as shown in Figure 2 (a). Many of the returned matches were incorrect and additional filtering of the matches was required. Figure 2 shows the sharp image on the left and a blurred image on the right, with blue lines show the connection between sharp and blurred feature points. Figure 2 demonstrates that increasing camera displacement influences both the number of feature points and correctness of feature matching. In (b) and (c) the connections are converging on a few features which must be incorrect because the blurred image is made with the same orientation and from the same position as the sharp image and shows the same scene. Filtering out the incorrect matches can be done using an approximate position and orientation of the images. In real UAV images this can be extracted from recorded GNSS and IMU data. Using the flight altitude and the camera perspective centre for both images the offset between the images can be calculated. By using this method it can be

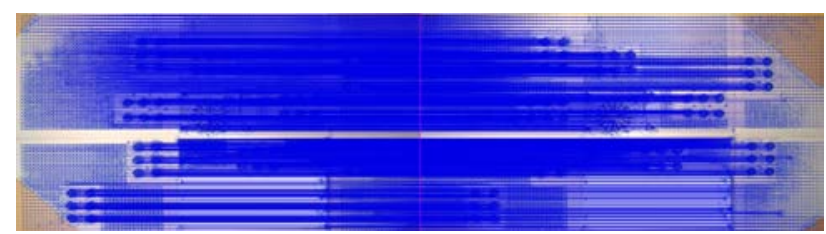

(a)

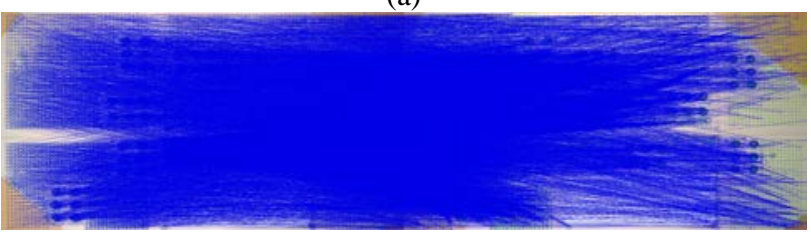

(b)

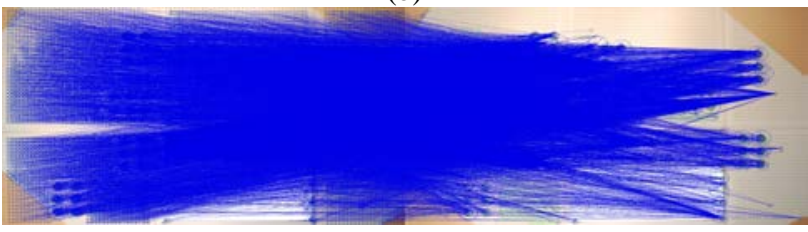

(c)

Figure 2. Influence of image blur on automatic feature detection and connection using SURF and brute force matching. Left image is a sharp reference image. Blue lines and circles indicate feature points (a) Right image without camera displacement. (b) Right image with $0.377 \mathrm{~mm}$ camera displacement. (c) Right image with $1.028 \mathrm{~mm}$ camera displacement. 


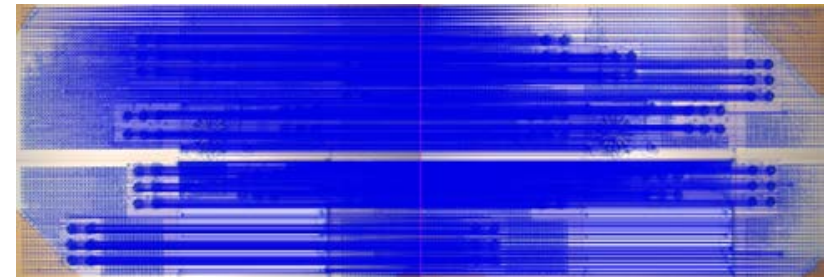

(a)

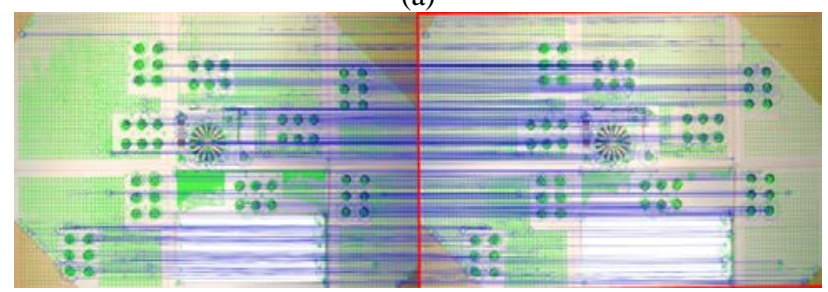

(b)

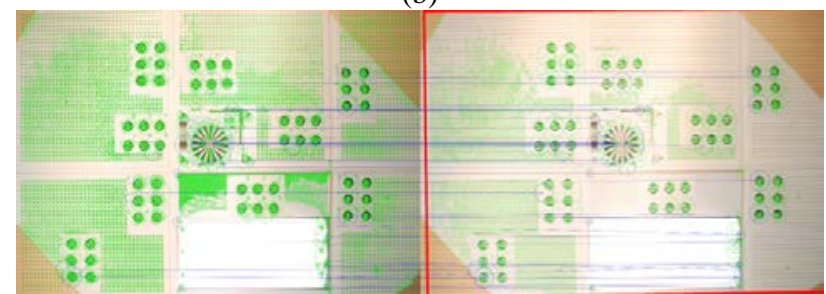

(c)

Figure 3. Results of automatic feature detection and connection using SURF and Brute Force matching after filtering out incorrect matches using sensor position. Left image is a sharp reference image. Blue lines and circles indicate connected feature points, green indicate detected feature points, red indicates image overlap. (a) Right image without camera displacement. (b) Right image with $0.377 \mathrm{~mm}$ camera displacement. (c) Right image with $1.028 \mathrm{~mm}$ camera displacement.

estimated at which position the matched feature should be in the blurred image. If the matched feature is found at a different position, this match can be assumed to be incorrect and ignored. If the feature was matched at the right position, the orientation of the feature has to match with the orientation given by the IMU. However, it is important to recognise that GNSS and IMU can only provide an approximate value for the translation and rotation of the images. This problem can be tackled by defining an appropriate threshold for the discrepancy between calculated and actual position, rotation and scale of the matched feature.

Figure 3 demonstrates the number of acceptably matched feature points, which is significantly less than the total matches made. These matches can be considered correct as the connection lines between sharp and blurred image are parallel. Even with this small number of matches it is still possible to calculate transformation parameters to detect the relative translation and rotation of sharp and blurred image. These transformation parameters are necessary to define the image relationship required to carry out standard photogrammetric measurements. If it is not possible to match enough features between the sharp and blurred image, feature detection can perhaps be improved by enhancing the blurred image.

\subsubsection{Improving Feature Detection}

Enhancing the blurred image can result in improved results for image processing and this does not require additional information. A fast, easy and reliable method to achieve this was investigated using an "Unsharp Mask Filter". This approach blurs the input image and then subtracts the input

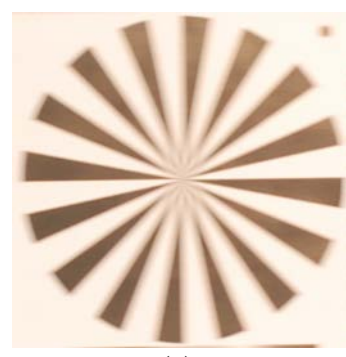

(a)

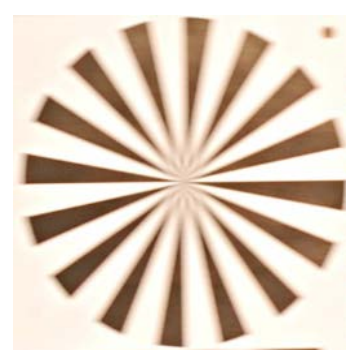

(b)
Figure 4. Figure (a) shows the blurred image before enhancement. (b) represents image (a) after enhancement. The image appears with stronger contrast but not sharp.

image from the result (GIMP, 2014). An enhanced image can be generated, which appears sharper than the original input image and with stronger contrast (Fig 4). Figure 4 represents a Siemens star to visualise the blur. Figure 4 (a) demonstrates that before enhancement it is easily possible to see the blur, especially in the centre of the star. Figure 4 (b) demonstrates that after enhancement the image appears sharper, but at the centre of the star it is possible to see that blur remains. The apparent higher contrast gives the impression of sharpness. However, with higher contrast it is possible that feature detection algorithms find feature points easier.

\subsection{Image Deblurring}

The effectiveness of initial feature detection, controls which image deblurring technique is best used. If the overlap calculation is successful, the information provided by the overlapping image can be used for deblurring. If the overlap is not successfully calculated, deblurring requires a different approach.

\subsubsection{Fourier Domain Approach}

A Fourier transformation can be adopted if points are successfully matched. As all images are taken from a moving platform it can be assumed that all images contain a certain amount of blur due to forward motion. However, some contain significantly more blur due to gusts, turbulences or operator inputs. If there is an overlap between a sharp and blurred image, then the overlap area in the blurred image can be deblurred.

In the first step the overlapping areas need to be transformed so that they have the same rotation, translation scale and shear. The parameters for this can be based on the results of SURF matching or manual feature detection and matching. After this the deblurring process can commence.

The frequency domain of the overlap is calculated for both images using a discrete Fourier transformation (DFT). The frequency domain of the more blurred image contains less high frequency elements than the sharper image. As both images show the same area, deblurring can be conducted by integrating the high frequencies of the sharper image into the frequency domain of the blurred image.

Transforming the frequency domain representation of the blurred image, (now enhanced with the high frequencies of the sharper image,) back to the spatial domain, then generates a less blurry image. As DFT is only possible for grey scale images it is necessary to either correct all channels separately or calculate a single grey scale representation. If a one channel grey scale image is used a final step transferring this back to a three channel colour image is required. The approach used is based 


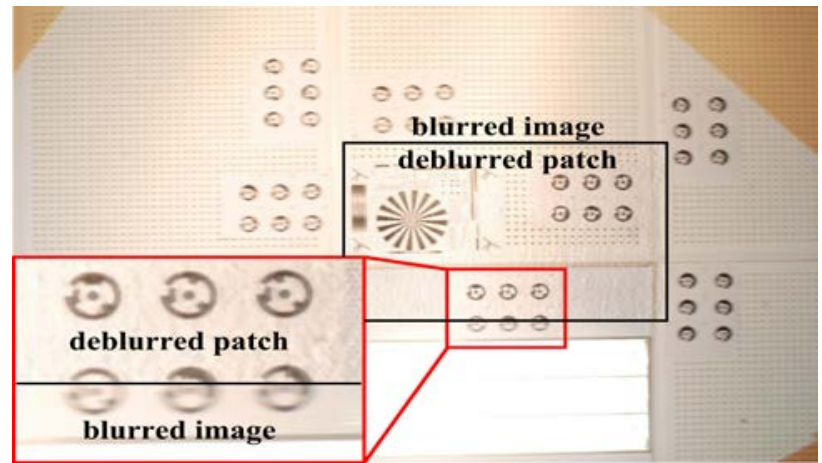

Figure 5. A deblurred patch is presented in the centre of the image. It is possible to see the photogrammetric targets are sharper and better to read and the boreholes in the ceiling panels are good to see. The inset shows a comparison between the deblurred upper part and the bottom part of a target which contains a camera displacement of $1 \mathrm{~mm}$ and visualizes how effective the deblurring is.

on the idea of pan sharpening by replacing the intensity channel of an image with the deblurred image (Laben, 2000).

After the deblurring process, the overlapping area needs to be transformed using an appropriate rotation, translation, scale and shear. As this deblurring is only possible for the overlapping area and not the complete image, the deblurred area of the image needs to replace the overlapping area in the blurred image. The result is a partially deblurred image (Fig 5).

\subsubsection{Edge Correction Approach}

Another approach developed in this study focused on correcting edges in images, which represent an important visual component. Edges indicate if an image is blurred or sharp (Chen, 2011) and help to identify how much an image is blurred. Also point spread functions and blur kernels can be calculated, based on edges or are part of additional information provided in other ways.

Knowing this, the image can be reduced to its edges by using a standard edge detection algorithm like a Laplace filter or similar. In a blurred image, the edges are displaced from their 'sharp' position due to motion of the camera during exposure (Fig 6 (b)). Also the intensity of the detected edge in a blurred image is much less than in a sharp image. This shift can be reversed by knowing the blur kernel so that the edge can be shifted back to its original position (Fig 6 (c)). How large the blur kernel is can be determined using overlapping images, IMU data or outputs of point spread calculation methods. Figure 6 (a) presents edges detected in a sharp image and Figure 6 (b) how the edges appear in a blurred image. The blurred edges are not round but oval. Figure 6 (c) is a re-sharpened image using the edge shift approach. It is possible to identify that the targets in the re-sharpened image are now circular in shape. Additionally, it is possible for the human brain to interpret elements of the original code. The errors which appear in this edge corrected image will be discussed further in the results section (4.2.2).

\section{RESULTS AND DISCUSSION}

The accuracy of the non-blind deconvolution methods used here depends upon which algorithm is used and the quality of the additional information. This additional information is dependent upon the feature detection and matching which is itself influenced by blur. It is important to reassess both, the feature detection and deblurring approaches used.

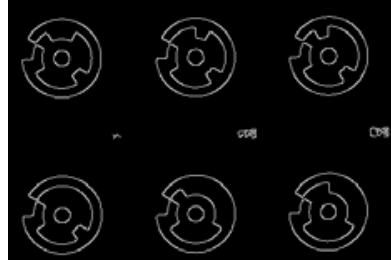

(a)

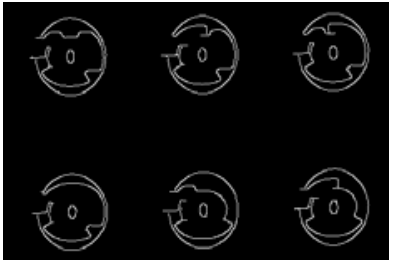

(b)

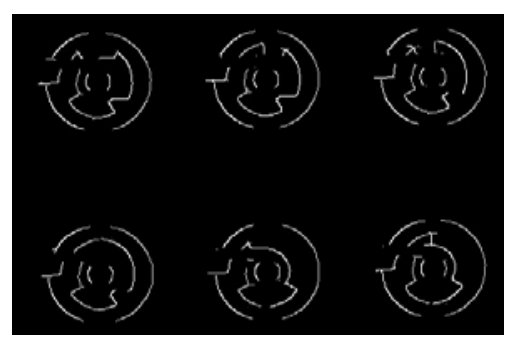

(c)

Figure 6. Figure (a) represents edges of circular targets in a sharp image. (b) shows the same edges in a blurred image. They do not appear as circles any longer. (c) is a preliminary result of the deblurring process based on the blurred edges (b) and knowledge about the blur path.

\subsection{Feature Detection}

As expected, it was found that with increasing blur the total number of detected feature points decreases (Table 1). For example, a camera displacement of $0.377 \mathrm{~mm}$ during exposure, results in just $72 \%$ of the original feature points being detected. Human hand jitter has frequencies of $2-10 \mathrm{~Hz}$ with an amplitude of up to $1 \mathrm{~mm}$ (Stiles, 1976) which is a likely cause for much motion and has to be considered as a significant influence.

The results after filtering out the incorrect matches using IMU and GNSS information, show that blur has an extreme influence on matching methods. Only a fraction of features were correctly connected between sharp and blurred image. In the case of a blurred image with a $1 \mathrm{~mm}$ displaced camera, only 47 matches were accepted (Table 1).

\begin{tabular}{lll}
\hline \hline $\begin{array}{l}\text { Camera } \\
\text { displacement [mm] }\end{array}$ & $\begin{array}{l}\text { Detected Feature } \\
\text { Points }\end{array}$ & $\begin{array}{l}\text { Accepted Feature } \\
\text { Points after Filtering }\end{array}$ \\
\hline 0.0 & $12214(100 \%)$ & $12214(100 \%)$ \\
0.377 & $8847(72 \%)$ & $1524(17 \%)$ \\
0.529 & $7370(60 \%)$ & $224(3 \%)$ \\
1.028 & $2645(22 \%)$ & $47(1.8 \%)$ \\
\hline \hline
\end{tabular}

Table 1. Impact of increasing image blur and feature detection. With increasing image blur the number of detected and accepted feature points reduces rapidly.

The feature detection shows that blur influences image processing, which confirms findings made by Sieberth et al. (unpublished). The more an image is blurred the fewer features are detected using SURF and the number of matched features becomes increasingly incorrect. However, the test images were taken with a short camera to object distance which questions the applicability for larger camera to object distances, which are around $100 \mathrm{~m}$ for typical UAV image flights. Therefore the camera displacement for a typical UAV image flight should be calculated. Normal UAV flight speed is supposed to be $54 \mathrm{~km} / \mathrm{h}$ and an image exposure time of 1/400 s (used by Grenzdörffer et. al (2012)), implies that the camera should experience a displacement of $37 \mathrm{~mm}$ during exposure. This is 70 times more 
than in the laboratory test with a displacement of $0.53 \mathrm{~mm}$. The camera to object distances in the lab test was $1.8 \mathrm{~m}, 55$ times shorter than normal UAV flight altitude of $100 \mathrm{~m}$. The ratios show that the lab tests are actually comparable with a typical UAV image flight because the camera displacement is as many times larger as the camera to object distance. Furthermore, this calculation does not consider that angular movements of UAVs which are much faster and cause more extensive image blur (Grenzdörffer et al., 2012). The test images also provide a texture with high contrast enabling SURF to find many feature points. SURF is the recommended method for UAV images (Gülch, 2012) but will experience problems with blurry images of low contrast areas (e.g. grass). Additionally, image overlap in the test image is nearly $100 \%$ but will be around only $60 \%$ in real scenes. This reduced overlap will reduce the feature points which are available in both images and reduces the chance for correct calculation of transformation parameters. In addition, the laboratory images do contain only linear movement without angular rotations. This simplifies the feature matching which will be significantly more difficult with more challenging camera displacements and rotations.

Enhancing the blurred image certainly improves the image. Instead of only 2645 features, twice as many features can be found and matched in an image with as much as $1 \mathrm{~mm}$ camera displacement. Out of these 5844 matched features 91 matches were accepted as correct, twice as many as before. This shows, that fast and easy to compute image enhancements can improve significantly the image quality necessary for photogrammetry.

\subsection{Deblurring}

Even if blur is insufficient to prevent successful image matching, it may be too severe for accurate detection and identification of photogrammetric targets and ground control points. A method to improve the image quality through "deblurring" is therefore valuable. Two approaches have been investigated.

\subsubsection{Fourier Approach}

The Fourier approach (Section 3.2.1) inserts high frequencies derived from a sharp image into the blurred and appeared successful (Fig 5). Figure 5 shows that photogrammetric targets in the deblurred patch can be identified easily and in comparison to targets in the remaining blurred part of the image, they are now sharp and have high contrast. A deblurred image for feature detection and matching is presented in Figure 7. The blurred image had a camera displacement of $0.53 \mathrm{~mm}$. After deblurring, SURF returns 11725 feature points. After matching and filtering incorrect features, 1124 were accepted as correct. This is five times more than with the original blurred image.

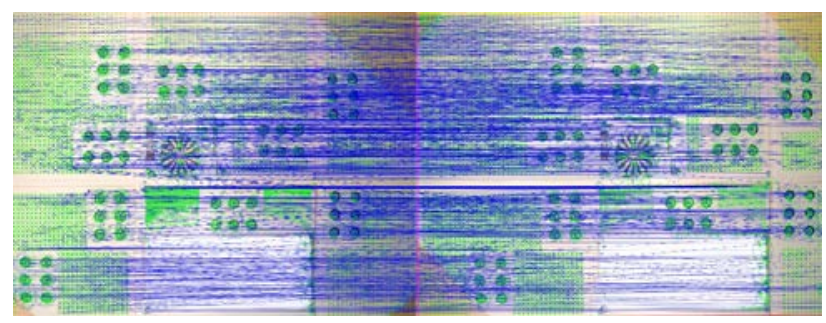

Figure 7. SURF feature detection applied on a deblurred image. On the right a sharp image. On the left a deblurred image which had a camera displacement of $0.53 \mathrm{~mm}$.
However, the approach using Fourier transformation is only applicable for flat areas, which do not exhibit significant height differences. In cases of significant height variations, like high rise buildings, tall trees or opencast pits, offsets will be generated due to relief displacement (Campbell and Wynne, 2012). A way to solve this is by using many small image patches, which take account for different heights.

Adding frequencies to a blurred image also causes some noise after transforming from the frequency back to the spatial domain. In the subsequent step of image transformation necessary to regain the correct position, rotation, shear and scale, the interpolation of the correct pixel intensities for the rectified image can cause a 'blurring' effect. Furthermore, deblurring using overlapping images acquired from a moving platform will only be as good as the 'sharper' image. If the overlapping image is completely sharp then the deblurred images will be sharp. If the overlapping image is slightly blurry the deblurred image will be blurry too because the critical high frequencies are missing and cannot be integrated to the blurred image. A complete deblurring can only be generated if there is a perfectly sharp image containing all high frequencies.

From a photogrammetric point of view this approach achieves images suitable for further processing. However, it cannot be guaranteed that subsequent measurements in the deblurred image are of high or even sub-pixel accuracy. Due to the geometric image transformation, errors can be introduced which depend on the accuracy of the transformation parameters. This shows the demand for a geometric correct approach, which guarantees correct photogrammetric measurements. This could be possible by using the edge shift approach.

\subsubsection{Edge Shifting Approach}

The edge shifting approach is a novel method developed here and shows promising results. The main problem is the disregard of colour information, which needs to be restored afterwards. This should be acceptable as the colour just needs to refill the shapes generated by the shifted edges. A more significant problem are the many special cases and exceptions, which can occur. Handling these exceptions is connected with a range of difficulties. It is possible that the shifting process generate holes in the shapes (Figure 6 (c)). This is caused by shifting two edges apart from one another and effectively 'ripping' them apart. Furthermore, edges which have completely disappeared cannot be recovered. The complexity of real images with different edge intensities, directions and blur length is much higher than in this high contrast test image.

However, deblurring of high contrast areas can also be usable for aerial images because aerial photogrammetric targets normally provide high contrast. It should be possible to achieve edge shifting with photogrammetric targets and get acceptable results which can be then accurately measured. Subsequent target measurements can return sub-pixel accuracies due to a sub-pixel precise shifting process. Furthermore, it might be possible to incorporate different blur in different positions of an image by calculating localised blur kernels. This would be necessary for blurred objects close to the camera, which appear more blurred compared to objects further away from the camera. Theoretically this approach is achievable and does not consume too much computational power. However, this approach is very complex due to the complexity and exceptions for shifting edges. It will be difficult to implement such an approach. 


\section{CONCLUSION}

This study has shown that image blur caused by camera displacement during image exposure has a clear and significant influence on photogrammetric processing. Successful feature detection and matching becomes increasingly difficult with increasing image blur. Enhancing a blurred image might improve feature detection at low computational cost and is easy to implement. Reversing image blur in a photogrammetrically correct and precise way is more difficult and cannot be solved with conventional deblurring methods. The described approach here using Fourier transformation returns good deblurred result for flat areas. However, the dependency on sharp images for deblurring makes this approach difficult to apply. Additionally, the unavailability of $3 \mathrm{D}$ object coordinates required to correct blur explicitly for varying camera to object distances, limits this approach. The problem of geometrically correct deblurring might be solvable using the edge shift approach. However, this approach will need further development.

\section{REFERENCES}

Agrawal, A., Xu, Y., Raskar, R., 2012. Multi-image deblurring. US Patent 8,229,244.

Bay, H., Tuytelaars, T., Gool, L. Van, 2006. Surf: Speeded up robust features, in: Computer Vision - ECCV 2006. Springer Berlin Heidelberg, pp. 404-417.

Brown, L.G., 1992. A survey of image registration techniques. ACM Computing Surveys, 24(4), pp. 325-376.

Campbell, J.B., Wynne, R.H., 2012. Introduction to Remote Sensing, Fifth ed. Guilford Press, New York.

Chen, C., Chen, W., Bloom, J. a., 2011. A universal referencefree blurriness measure. Society of Photo Optical Instrumentation Engineers. 7867(78670B-2), pp. 1-14.

Eisenbeiß, H., 2009. UAV photogrammetry. ETH Zürich, Zürich.

Eisenbeiß, H., 2011. Tutorial UAV-g Introduction. UAV-G. 2011.

GIMP, 2014. Unsharp Mask. http://docs.gimp.org/en/plug-inunsharp-mask.html (20 Jun. 2014).

Grenzdörffer, G., Niemeyer, F., Schmidt, F., 2012. Development of Four Vision Camera System For A MicroUAV. International Archives of the Photogrammetry, Remote Sensing and Spatial Information Sciences, XXXIX-B1, pp. 369-374.

Gülch, E., 2012. Photogrammetric Measurements in Fixed Wing UAV Imagery. International Archives of the Photogrammetry, Remote Sensing and Spatial Information Sciences, XXXIX-B1, pp. 381-386.

Joshi, N., Szeliski, R., Kriegman, D.J., 2008. PSF estimation using sharp edge prediction. IEEE Conference on Computer Vision and Pattern Recognition. pp. 1 - 8.

Kraus, K., 2004. Photogrammetrie, 7th ed. Walter de Gruyter, Berlin.

Kraus, K., 2007. Photogrammetry - Geometry from Images and Laser Scans, Second ed. Walter de Gruyter, Berlin.
Laben, C.A., Brower, B.V., 2000. Process for Enahncing the Spatial Resolution of Multispectral Imagery Using PanSharpening. US Patent 6,011,875.

Lelégard, L., Delaygue, E., Brédif, M., Vallet, B., 2012. Detecting and correcting motion blur from images shot with channel-dependent exposure time. International Annals of the Photogrammetry, Remote Sensing and Spatial Information Sciences, I-3, pp. 341-346.

Lowe, D.G., 2004. Distinctive Image Features from ScaleInvariant Keypoints. International Journal of Computer Vision, 60(2), pp. 91-110.

Lucy, L.B., 1974. An iterative technique for the rectification of observed distributions. Astronomical Journal, 79, pp. 745-754.

Luhmann, T., Robson, S., Kyle, S., Harley, I., 2006. Close Range Photogrammetry. Whittles Publishing.

OpenCV Developer Team, 2013. OpenCV API Reference OpenCV 2.4.6.0 http://docs.opencv.org/modules/refman.html (20 Jun. 2014)

Raskar, R., Agrawal, A., Tumblin, J., 2006. Coded exposure photography: motion deblurring using fluttered shutter. ACM Transactions on Graphics, 25(3) , pp. 795-804.

Richardson, W.H., 1972. Bayesian-Based Iterative Method of Image Restoration. Journal of the Optical Society of America, 62(1), pp. 55-59.

Shan, Q., Jia, J., Agarwala, A., 2008. High-quality motion deblurring from a single image. ACM Transactions on Graphics, 27(1), pp. 1-10.

Sieberth, T., Wackrow, R., Chandler, J.H., 2013. Automatic Isolation of Blurred Images from UAV Image Sequences. International Archives of the Photogrammetry, Remote Sensing and Spatial Information Sciences, XL-1/W2, pp. 361-366.

Sieberth, T., Wackrow, R., Chandler, J.H., unpublished. Blur Disturbs - Influence of Blurred Images in Photogrammetry. Submitted to The Photogrammetric Record.

Stiles, R.N., 1976. Frequency and displacement amplitude relations for normal hand tremor. Journal of Applied Physiology, 40(1), pp. 44 - 54.

Tai, Y.-W., Du, H., Brown, M.S., Lin, S., 2008. Image/video deblurring using a hybrid camera, IEEE Conference on Computer Vision and Pattern Recognition. pp. 1-8.

Wiener, N., 1949. Extrapolation, Interpolation, and Smoothing of Stationary Time Series. Wiley, New York.

\section{ACKNOWLEDGEMENTS}

I want to thank the RSPSoc for sponsoring my attendance at this conference. 